\title{
Impact of Stinging Jellyfish Proliferations along South Italian Coasts: Human Health Hazards, Treatment and Social Costs
}

Antonella De Donno ${ }^{1,2, *}$, Adele Idolo ${ }^{1,2}$, Francesco Bagordo ${ }^{1}$, Tiziana Grassi ${ }^{1}$, Alessandro Leomanni ${ }^{1}$, Francesca Serio ${ }^{1}$, Marcello Guido ${ }^{1}$, Mariarita Canitano ${ }^{3}$, Serena Zampardi ${ }^{1,2}$, Ferdinando Boero ${ }^{1,2,4}$ and Stefano Piraino ${ }^{1,2 *}$

1 Department of Biological and Environmental Sciences and Technologies (DiSTeBA), University of Salento, Lecce 73100, Italy; E-Mails: adele.idolo@unisalento.it (A.I.); francesco.bagordo@unisalento.it (F.B.); tiziana.grassi@unisalento.it (T.G.); alessandro.leomanni@unisalento.it (A.L.); francesca.serio@unisalento.it (F.S.); marcello.guido@unisalento.it (M.G.); serena.zampardi@unisalento.it (S.Z.); boero@unisalento.it (F.B.); stefano.piraino@unisalento.it (S.P.)

2 National InterUniversity Consortium for Marine Sciences (CoNISMa), Rome 00198, Italy

3 Local Health Authority of Lecce (ASL/LE), Lecce 73100, Italy; E-Mail: dirsan@ausl.le.it

4 Institute of Marine Sciences, National Research Council (ISMAR-CNR), Genoa 16149, Italy

* Authors to whom correspondence should be addressed;

E-Mails: antonella.dedonno@unisalento.it (A.D.); stefano.piraino@unisalento.it (S.P.); Tel.: +39-0832-298-687 (A.D.); +39-0832-298-616 (S.P.); Fax: +39-0832-298-626 (A.D.\&S.P.).

Received: 17 January 2014; in revised form: 9 February 2014 / Accepted: 12 February 2014 /

Published: 27 February 2014

Abstract: Stinging jellyfish outbreaks represent a health hazard, causing contact dermatitis and systemic reactions. This study investigated the epidemiology, severity, and treatment protocols of jellyfish stings in a coastal area with high tourist development and frequent stinging jellyfish outbreaks of the central Mediterranean (Salento, Southern Italy), and the associated costs for the Italian National Health Service. In 2007-2011, 1,733 bathers (mostly children and females) sought medical assistance following jellyfish stings, the main cause of human pathologies due to contact with marine organisms. The majority of events were reported in the years 2007-2009, whereas the occurrence of cnidarian jellyfish outbreaks has been increasingly reported in the same area since summer 2010 . Most symptoms were limited to local and cutaneous reactions; conversely, $8.7 \%$ of cases evoked complications, mainly due to allergic reactions. The main drugs used were 
corticosteroids, locally applied and systemic (46\% and 43\%, respectively), and with ammonia $(74 \%)$ as the main non-pharmacological treatment. The estimated cost of jellyfish-related first-aid services along the Salento coastline over the 5-year period was approximately 400,000 Euros. Therefore the management of jellyfish outbreak phenomena need coordinated research efforts towards a better understanding of underlying ecological mechanisms, together with the adoption of effective prevention policy, mitigation strategies, and appropriate planning of health services at tourist hot spots.

Keywords: jellyfish blooms; sting epidemiology; treatment protocols; Mediterranean Sea

\section{Introduction}

Interest in jellyfish grew considerably in recent years as a result of "anomalous" proliferations seen with increasing frequency in all seas and the new appearance of invasive species in temperate seas [1], linked to multiple causes such as climate change, overfishing and pollution [2-4]. Variations in temperature and salinity have been linked to variations in jellyfish abundance in a number of studies [5-8]. There is widespread concern that the oceans may increasingly be dominated by jellyfish, because many of them are able to increase in abundance rapidly and adapt to new conditions following ecosystem regime shifts [9]. Jellyfish include a polyphyletic invertebrate assemblage, mainly composed by cnidarian medusae and colonial siphonophores, ctenophores, pelagic tunicates (larvaceans, salps and doliolids), chaetognaths, polychaetes and other non-crustacean soft-bodied planktonic organisms. Jellyfish outbreaks (or blooms) can have important impacts on human coastal activities, such as fishing and leisure activities, but they can also represent a significant hazard to public health [10,11]. Indeed, cnidarian jellyfish are characterised by the possession of highly specialized mechano-receptor cell types in the animal kingdom: cnidocytes or stinging cells. These are used for prey capture and defense from predators by injection of venoms, represented by variety of proteinaceous and non-proteinaceous compounds, which may have cytotoxic, cytolytic and enzymatic properties [10,12-14]. A minority of cnidarian venoms, from some infamous "stinging jellyfish", are known to produce harmful toxic effects on humans $[14,15]$. Human envenomations by cnidarian toxins may produce immediate toxicity (from minutes to few hours), in combination with immediate or delayed allergic responses, determining local symptoms (dermatitis, oedema and swelling, itching, stiffness, necrosis, pain) or more severe systemic effects, including neurotoxic, cardiovascular, motory and respiratory problems, as well as anaphylaxis or anaphylactoid syndromes $[10,15]$.

Some of the most popular treatments commonly adopted to provide relief from jellyfish stings include vinegar, baking soda slurry, ammonia, and ethanol. Many of these chemicals are thought to inactivate undischarged cnidocysts (stinging capsules in the cnidocytes) on the skin so that further stinging is prevented. However, nematocyst discharge of various species of jellyfish has been reported upon treatments with alcohol, acetic acid, and urea [16,17]. Topical application of anaesthetics, e.g., benzocaine and lidocaine, is used to bring relief from jellyfish stings [16,18]. Even local application of corticosteroids or antihistamines relieves the pain, burning and redness [19]. Recently, Cegolon et al. [15] provided an excellent account of the available scientific information on jellyfish 
stings and their clinical management, highlighting that the venom specificity would require the adoption of species- or genus-specific treatment protocols, instead of a single generalized procedure, and that further research is required to validate the weak evidence of successful treatments. A species-specific treatment protocol has been recently adopted by the ENPI-CBC MED European research project MEDJELLYRISK, recommending specific treatment protocols for five common stinging jellyfish in the Mediterranean Sea [20].

Identification of the responsible jellyfish species is becoming easier in coastal areas where jellyfish monitoring programmes are commonly implemented. Jellyfish abundance and distribution in the Mediterranean Sea are regularly monitored since the late 1970s and early 1980s as abnormal outbreaks of several species occurred [11,21]. The largest blooms are mainly due to the schyphozoan Pelagia noctiluca, bearing a strong envenomation potential [22]. Besides $P$. noctiluca, other venomous jellyfish occur in the Mediterranean Sea: the hydrozoan Physalia physalis and P. utricularis, the cubozoan Carybdea marsupialis, the scyphozoan Chrysaora hysoscella, and Rhopilema nomadica, a stinging exotic jellyfish progressively spreading in the Mediterranean Sea from the Suez Canal [23,24]. As a whole, data concerning jellyfish impact on human health in the Mediterranean sea are scarce.

Few epidemiological studies of jellyfish stings have been carried out in Adriatic localities, while data from other Mediterranean regions are sporadic [19,25-31]. The aim of the present study was to investigate the epidemiology, severity and treatment of jellyfish stings over summer seasons across five years (2007-2011) in the Salento area (Southern Italy), their socio-economic impacts and policy implications for the Italian Health Service, with a preliminary comparison with the available information on jellyfish abundances gathered from an ongoing citizen science jellyfish monitoring campaign.

\section{Materials and Methods}

The study was performed in collaboration with the Local Health Authority of Lecce (ASL/LE) and involved collection and analysis of data from patients registered yearly at medical first-aid stations due to jellyfish envenomation in summer (July and August) 2007-2011. The data included two emergency ambulances, four hospitals, and twenty-one summer first aid centres (set up by ASL/LE in the summer months and managed by the relevant Health Districts) along the coast of the Salento peninsula, Italy (Figure 1).

The study area has a resident population of over 815,500 inhabitants that increased by about an additional $9 \%$ in the summer period by tourists [32]. The peninsula is bounded by the Ionian and Adriatic seas with a coastline of $215 \mathrm{~km}$, characterized by rocky coasts $(156.5 \mathrm{~km} ; 73 \%)$ and sandy beaches $(58.5 \mathrm{~km} ; 27 \%)$. The study area was divided into three coastal zones:

1. Adriatic Coast, $62 \mathrm{~km}$ long;

2. Lower Ionian Coast, $105 \mathrm{~km}$ long;

3. Upper Ionian Coast, $48 \mathrm{~km}$ long.

For each patient an anonymous report was compiled with information regarding the date and time of the assistance provided, the place where the patient was stung by jellyfish, demographic data (age, sex, provenance) and clinical information (site and type of lesion, symptoms, therapeutic methods, complications). 
Figure 1. Health centres along the coast of the Salento peninsula (Italy). (\# = Summer first aid centre, $\mathbf{H}=$ Hospital, $=$ emergency ambulance).

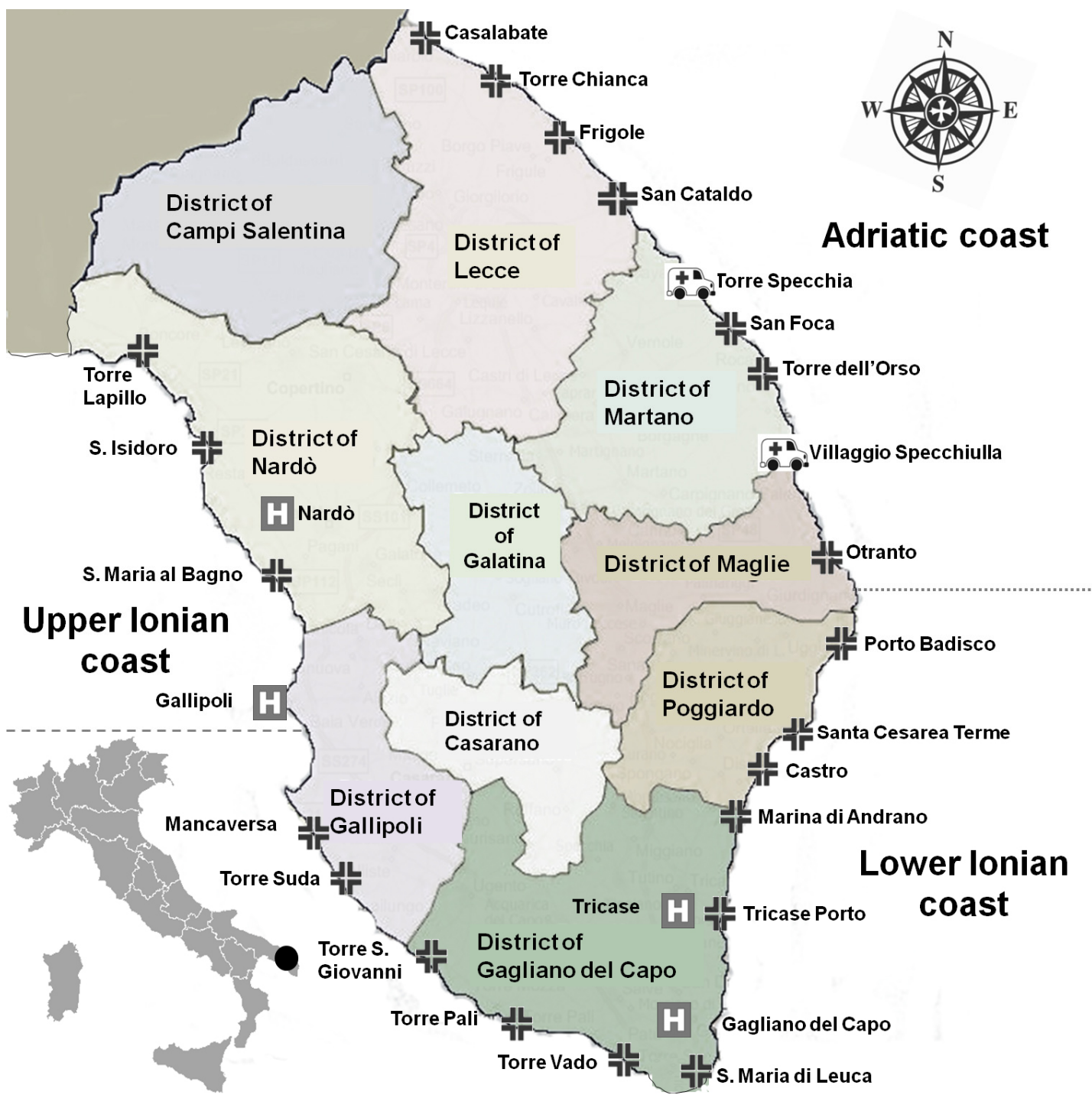

The incidence of treatments provided for jellyfish envenomations with respect to the total number of visits at summer first aid centres was calculated. Data were analyzed as semimonthly sets: 1 st -15 th, 16th-31st July and August. Patients receiving care were grouped by age: 1-10, 11-20, $21-30,31-40,41-50,51-60,>60$ years. The subjects were subdivided into three groups of geographical provenance: "local residents", "Italian tourists" and "foreign tourists".

Data on predominant wind directions for the period of investigation were obtained from the Laboratory of Climatology, Department of Materials Science, University of Salento, in order to correlate jellyfish occurrence and wind-driven currents,.

The economic cost of basic first aid treatments afforded by the Italian Health Service (SSN) was extracted from guidelines set out in the national and regional regulations [33]. In Italian accident and emergency (A\&E) wards, the triage system is applied according to colour codes denoting the urgency of treatment. This color-coding scheme uses four classes in order of increasing severity: white, green, yellow and red. Each jellyfish envenomation treatment at the summer first aid centres 
was assigned "code white" treatment (less serious, low priority case), entailing an average expense of $€ 226.00$ [33].

Data on jellyfish records along the Salento coasts during the years 2009-2011 were obtained by the dataset of the METEOMEDUSE project, conceived by the University of Salento, Lecce, and endorsed by the popular science magazine FOCUS. Thousands of records from the Italian coastlines were sent through short-message-service (SMS) messages, e-mails, internet forms, or by using a dedicated freely downloadable application for the iPhone operating system (IOS) and Android smartphones (https://itunes.apple.com/en/app/focus-meteo-meduse/id445832425?mt=8).

\section{Results}

In the study area 1,733 bathers sought medical assistance following contact with jellyfish in the summer months of the period 2007-2011. The majority of cases occurred in the first three years: $25.7 \%$ (446 cases) of total jellyfish stings were reported in summer 2007, $22.5 \%$ (390) in 2008, 24.7\% (427) in 2009, whereas a reduction of the incidence of jellyfish stings was observed in $2010(17.6 \%)$ and 2011 (9.5\%) (Table 1), with a mean of 6.9 bathers/day requiring medical assistance in 2009 to 2.7 bathers/day in 2011. In parallel, increasing jellyfish outbreaks were recorded from 2009 to 2011 along the coasts of Salento by means of the citizen science campaign METEOMEDUSE (Figure 2). More generally, 2,344 records of stinging jellyfish were documented from 2009 to 2011 along Italian coasts (Figure 3). In the study area, the most common jellyfish stingers were the scyphozoan Pelagia noctiluca (61.81\%), the cubozoan Carybdea marsupialis (26.57\%); occasionally, the hydrozoan Olindias phosphorica (6.48\%), and the scyphozoan Chrysaora hysoscella were locally abundant (5.11\%). The scyphozoan Cotylorhiza tuberculata was also very common, but the stinging potential of this species is almost negligible.

Jellyfish stings in the Salento peninsula were one of the most frequent reasons for people seeking health assistance in summer (9th after accidents, mycosis, vomiting, insect bites, otitis, renal colic, panic, congestion), accounting for about $3 \%$ of total visits at summer first aid stations. However, jellyfish stings were the main cause of human pathologies resulting from contact with marine organisms.

The spatial and temporal distribution showed a consistent pattern in all five bathing seasons studied. $65 \%$ of cases $(1,138)$ were recorded on the Adriatic coast, with just 19\% (326) on the Upper Ionian Coast and 16\% (269) on the Lower Ionian Coast. In the period of observation an average of $8.1 \mathrm{cases} / \mathrm{km}$ of coastline was recorded, ranging from a maximum of $18.4 \mathrm{cases} / \mathrm{km}$ along the Adriatic coast to a minimum of 2.6 cases $/ \mathrm{km}$ on the Lower Ionian. 
Table 1. Number of cases of jellyfish stings reported at health centres along the coast of the Salento peninsula (Italy) in the period 2007-2011 (SFAC: Summer First Aid Centre; H: Hospital; EA: Emergency Ambulance).

\begin{tabular}{|c|c|c|c|c|c|c|c|}
\hline \multirow{3}{*}{ Location } & \multirow{3}{*}{ Health Centre } & \multicolumn{6}{|c|}{ No. of Cases Reported } \\
\hline & & & & & & & TOTAL \\
\hline & & 2007 & 2008 & 2009 & 2010 & 2011 & 2007-2011 \\
\hline \multicolumn{8}{|l|}{ ADRIATIC COAST } \\
\hline Casalabate & SFAC & 6 & 35 & 27 & 18 & 8 & 94 \\
\hline Torre Chianca & SFAC & 69 & 72 & 50 & 23 & 14 & 228 \\
\hline Frigole & SFAC & inactive & inactive & 11 & 26 & 20 & 57 \\
\hline San Cataldo & SFAC & 63 & 71 & 43 & 103 & 19 & 299 \\
\hline Torre Specchia & EA & 0 & 3 & 0 & 0 & 0 & 3 \\
\hline San Foca & SFAC & 73 & 31 & 65 & 10 & 11 & 190 \\
\hline Torre dell'Orso & SFAC & 62 & 26 & 59 & 7 & 6 & 160 \\
\hline Villaggio Specchiulla & EA & 0 & 1 & 0 & 0 & 0 & 1 \\
\hline Otranto & SFAC & 56 & 18 & 18 & 3 & 11 & 106 \\
\hline Total cases along the Adriatic coast & & 329 & 257 & 273 & 190 & 89 & 1,138 \\
\hline \multicolumn{8}{|l|}{ LOWER IONIAN COAST } \\
\hline Porto Badisco & SFAC & inactive & inactive & 3 & 5 & 1 & 9 \\
\hline Santa Cesarea Terme & SFAC & 17 & 8 & 10 & 9 & 2 & 46 \\
\hline Castro & SFAC & 1 & 3 & 8 & 1 & 2 & 15 \\
\hline Marina di Andrano & SFAC & 0 & 1 & 6 & 5 & 1 & 13 \\
\hline Tricase & $\mathrm{H}$ & 0 & 0 & 0 & 0 & 0 & 0 \\
\hline Tricase Porto & SFAC & 7 & 0 & 0 & 0 & 2 & 9 \\
\hline Gagliano del Capo & $\mathrm{H}$ & 1 & 0 & 0 & 0 & 0 & 1 \\
\hline S. Maria di Leuca & SFAC & 1 & 4 & 3 & 0 & 2 & 10 \\
\hline Torre Vado & SFAC & 10 & 5 & 12 & 0 & 5 & 32 \\
\hline Torre Pali & SFAC & 0 & inactive & inactive & 10 & 5 & 15 \\
\hline Torre San Giovanni & SFAC & 3 & 4 & 9 & 6 & 11 & 33 \\
\hline Torre Suda & SFAC & 12 & 7 & 13 & 10 & 2 & 44 \\
\hline Mancaversa & SFAC & 7 & 8 & 16 & 10 & 1 & 42 \\
\hline Total cases along the lower Ionian coast & & 59 & 40 & 80 & 56 & 34 & 269 \\
\hline \multicolumn{8}{|l|}{ UPPER IONIAN COAST } \\
\hline Gallipoli & $\mathrm{H}$ & 7 & 9 & 21 & 0 & 2 & 39 \\
\hline Santa Maria al Bagno & SFAC & 4 & 10 & 12 & 13 & 4 & 43 \\
\hline Nardò & $\mathrm{H}$ & 1 & 0 & 0 & 3 & 0 & 4 \\
\hline S. Isidoro & SFAC & 24 & 16 & 15 & 33 & 16 & 104 \\
\hline Torre Lapillo & SFAC & 22 & 58 & 26 & 10 & 20 & 136 \\
\hline Total cases along the upper Ionian coast & & 58 & 93 & 74 & 59 & 42 & 326 \\
\hline \multicolumn{8}{|l|}{ Total cases along the entire coast of the } \\
\hline Salento & & 446 & 390 & 427 & 305 & 165 & 1,733 \\
\hline
\end{tabular}


Figure 2. Jellyfish occurrence along the Salento coastline (2009-2011).

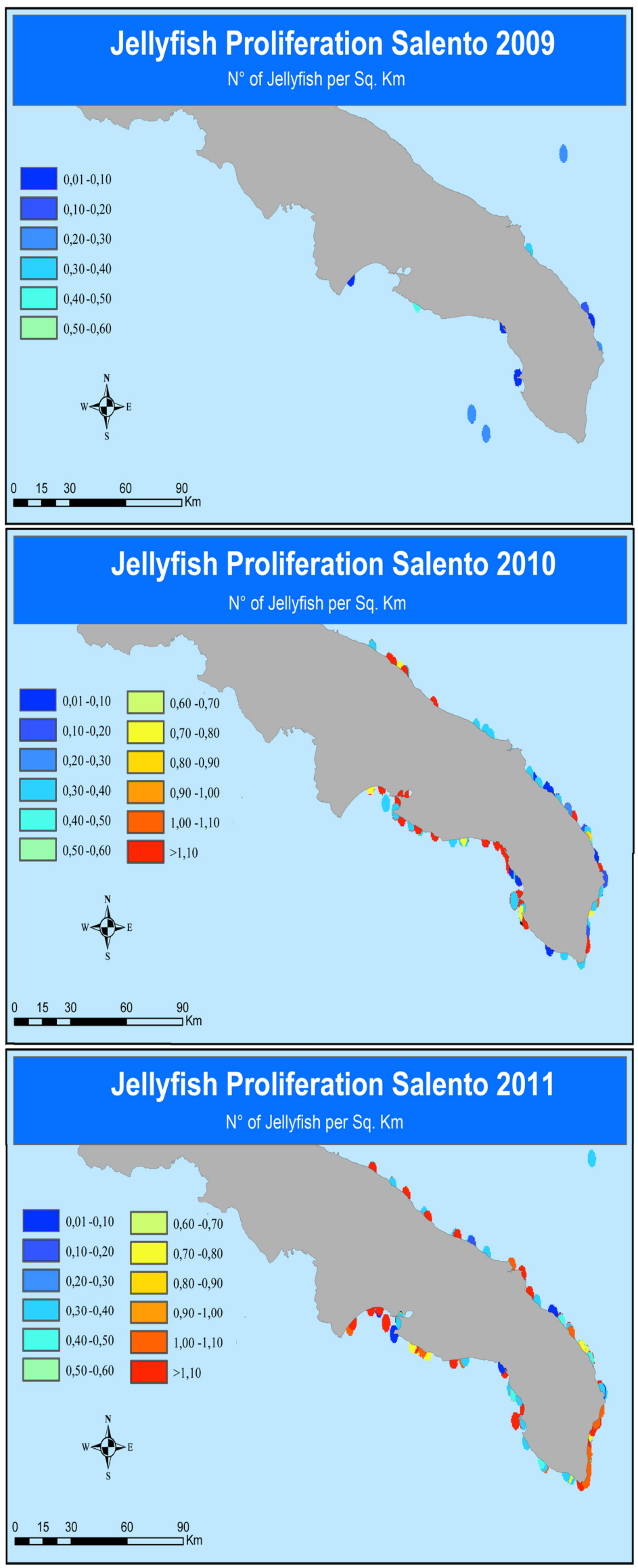


Figure 3. Number of citizen science records of the most common stinging jellyfish along Italian coasts (2009-2011).

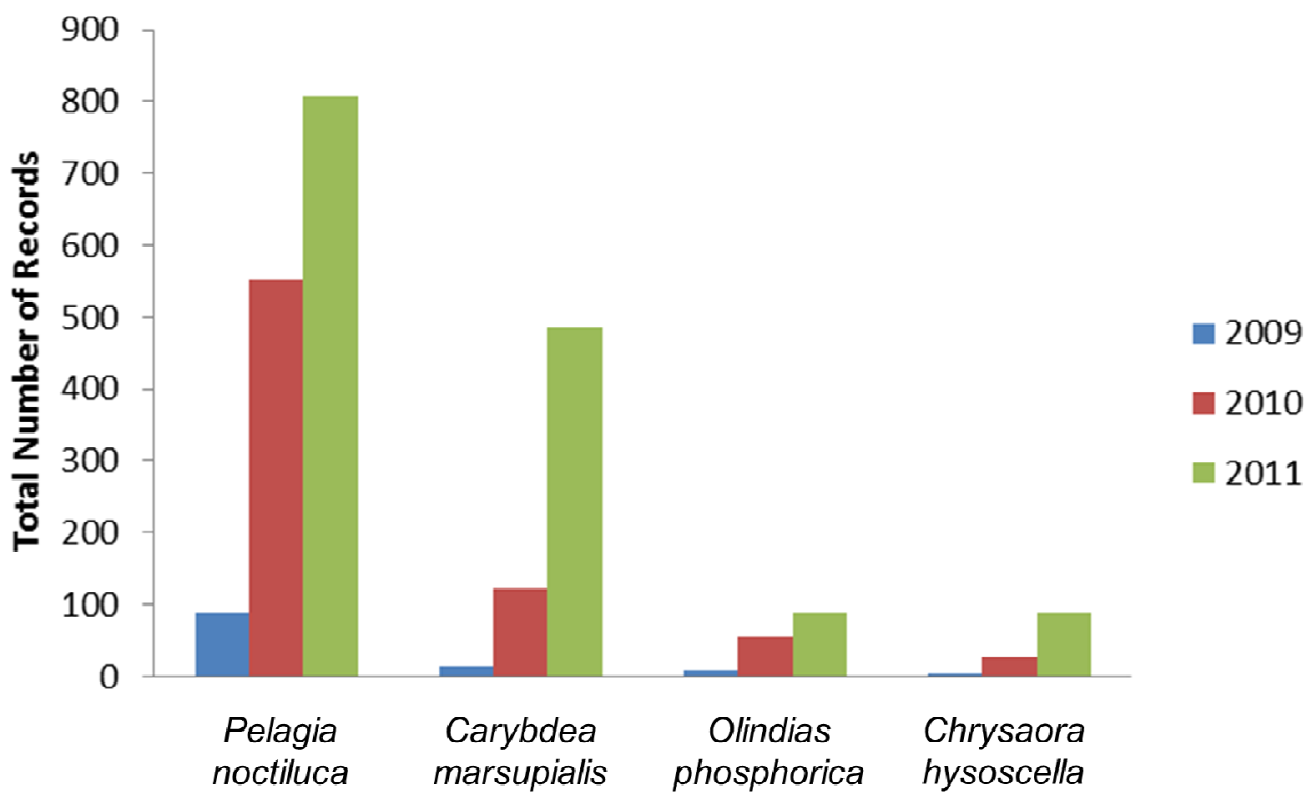

As expected, a temporal trend of incidence was observed, highlighting a peak in jellyfish stings in August, which accounted for 1,194 cases (68.9\%). Along the Adriatic coast, the frequency of cases increased over the summer period, from $4.6 \%$ (80) in the first half of July to $27.6 \%$ (479) in the second half of August. In contrast, along the Upper and Lower Ionian Coasts the incidence of stings was constantly low (Figure 4).

Figure 4. Spatial and temporal distribution of cases of jellyfish stings.

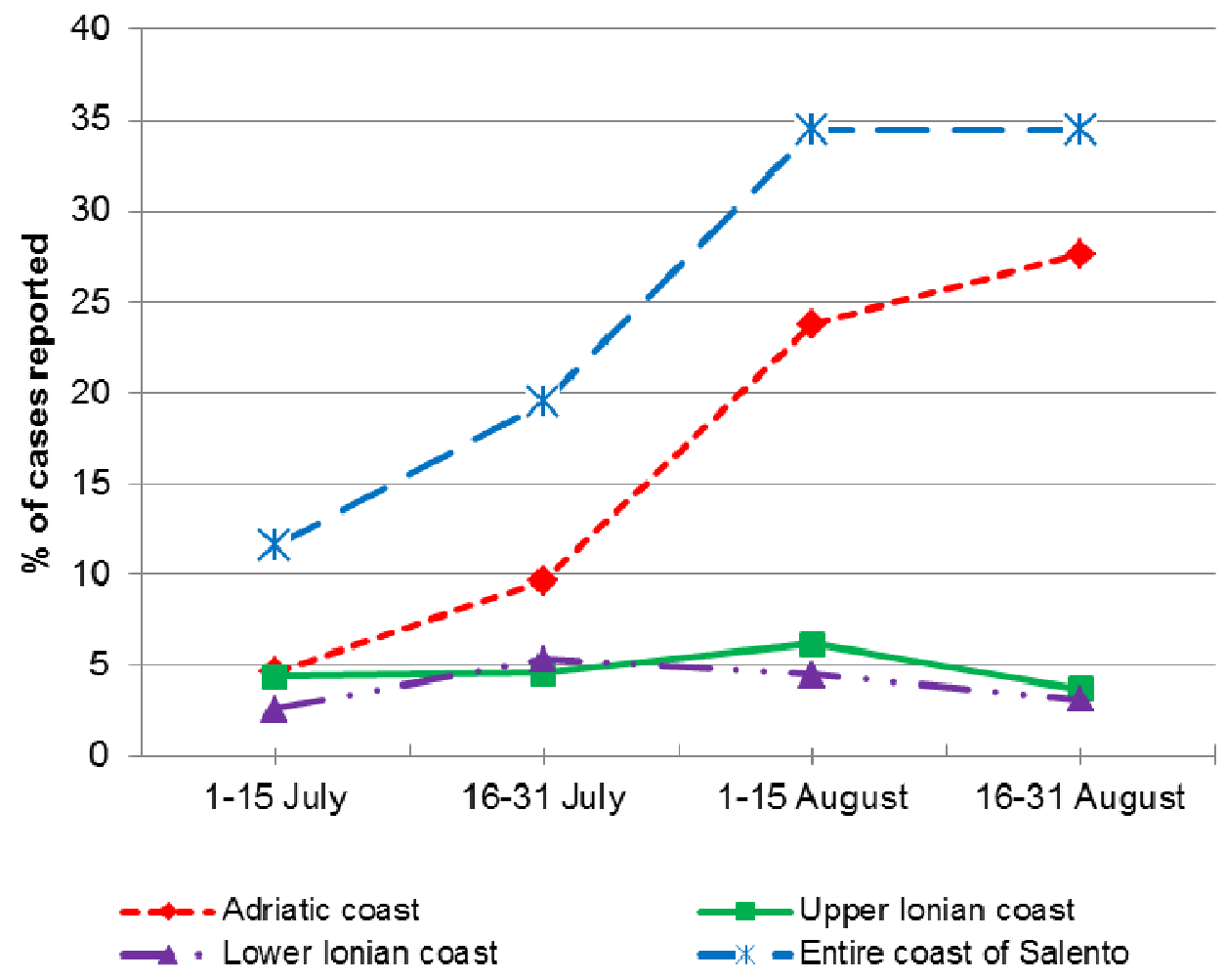


Data on wind direction showed a predominance of northerly winds throughout the study period (242/310 days). This wind pattern generates currents directed towards the Italian Adriatic Sea coastline and away from the Ionian Sea coast. High concentration of jellyfish in shallow waters (as in proximity of sandy beaches) is mostly observed when winds blow perpendicularly to the coast.

In general, patients seeking for medical assistance at the surveyed summer A\&E centers were aged over $60(33 \%)$, with a small majority of males (52\%) and Italian tourists (53\%). However, jellyfish stings occurred most frequently in younger bathers, especially belonging to the 1-10 year age group (28.6\%), and the 11-20 year age group (24.7\%). The proportion of stings accounted for by individuals aged between 21 and 30 and 31 and 40 was remarkably lower (16.9\% and 10.3\%, respectively). Other age groups were affected less frequently (Figure 5).

Figure 5. Distribution of cases of jellyfish stings in relation to patient gender (M, male; $\mathrm{F}$, female) and age.

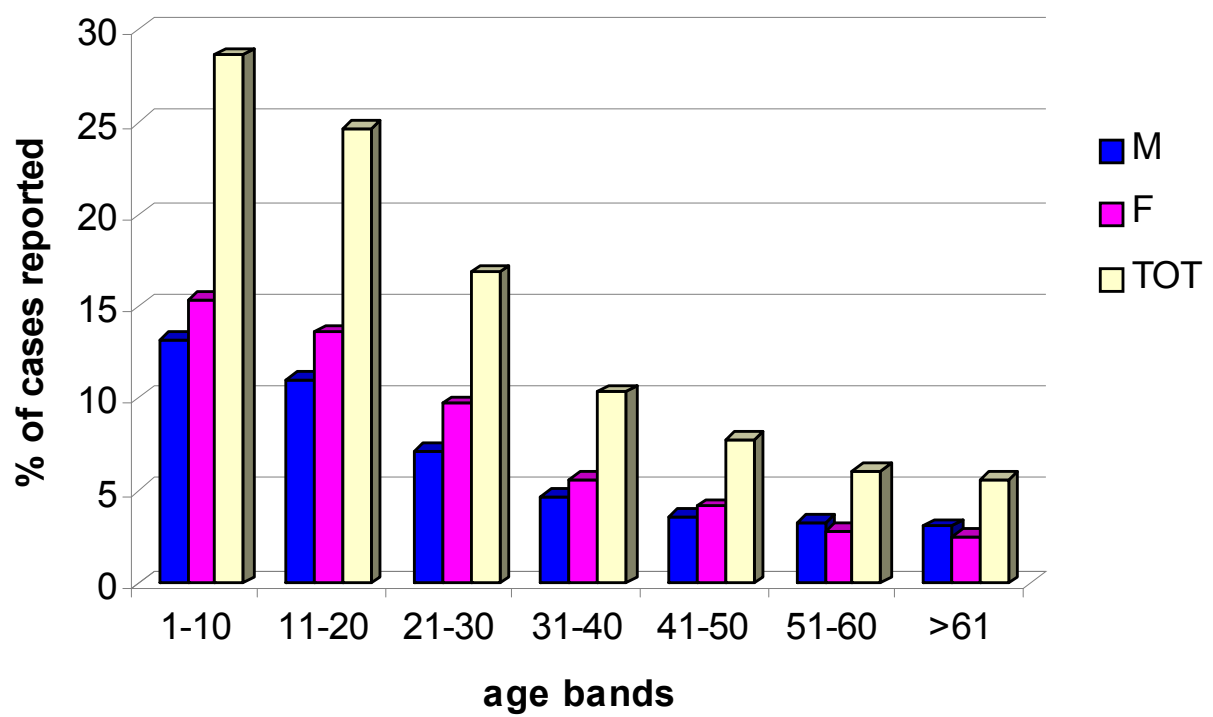

Regarding gender, a slim majority of stung bathers were female $(53.3 \%)$, with the greatest differences seen in subjects up to 30 years old. The data on the provenance of the subjects showed that $62 \%$ of the individuals seeking treatment were local residents, $35 \%$ of cases were tourists from other areas of Italy, and 3\% were foreign tourists.

Regarding parts of the body affected by stings, in most cases, the stings were on the lower limbs (41\%) and upper limbs (23\%). Many subjects also sought medical assistance for stings to the face $(17 \%)$ and for "multiple stings" $(13 \%)$, resulting from contact with jellyfish tentacles on various parts of the body. Stings to the thorax, abdomen and back accounted for only a few cases $(3 \%, 2 \%$ and $1 \%$, respectively) (Figure 6).

The most frequent symptoms occurring after jellyfish stings were local and cutaneous reactions (redness, pain, itching, intense burning, vesicles). In $8.7 \%$ of cases, however, there were complications, mainly allergic reactions $(6.2 \%)$. The other complications included ocular oedema $(1.3 \%)$, urticaria $(0.5 \%)$, conjunctivitis $(0.2 \%)$, panic attacks $(0.2 \%)$, dyspnea $(0.1 \%)$, muscular spasms $(0.1 \%)$, infection $(0.1 \%)$, and one case of anaphylactic shock $(0.1 \%)$. 
The treatments applied are presented in Figure 7. As can be seen, locally applied and systemic corticosteroids are the most frequently used drugs ( $46 \%$ and $43 \%$, respectively). Other medicaments used were systemic and locally applied antihistamines, specific treatments, anti-inflammatory agents or analgesics and non-pharmacological treatments. The latter mainly consisted of rinsing with liquid ammonia (74\%), physiological saline solution (12\%), alcohol (9\%), chlorine-based disinfectants (7\%), hot water $(7 \%)$, cold water $(3 \%)$ or application of ice $(1 \%)$, used individually or combined between them.

Figure 6. Parts of the body affected by jellyfish stings.

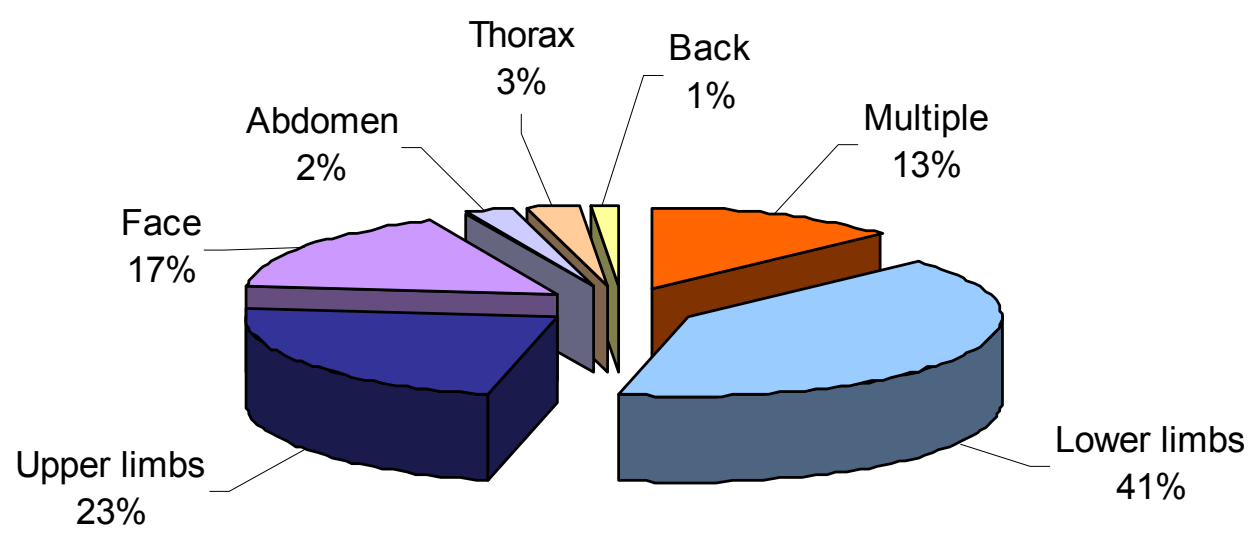

Figure 7. Medical treatments for jellyfish stings applied at first aid centres in the Salento peninsula.

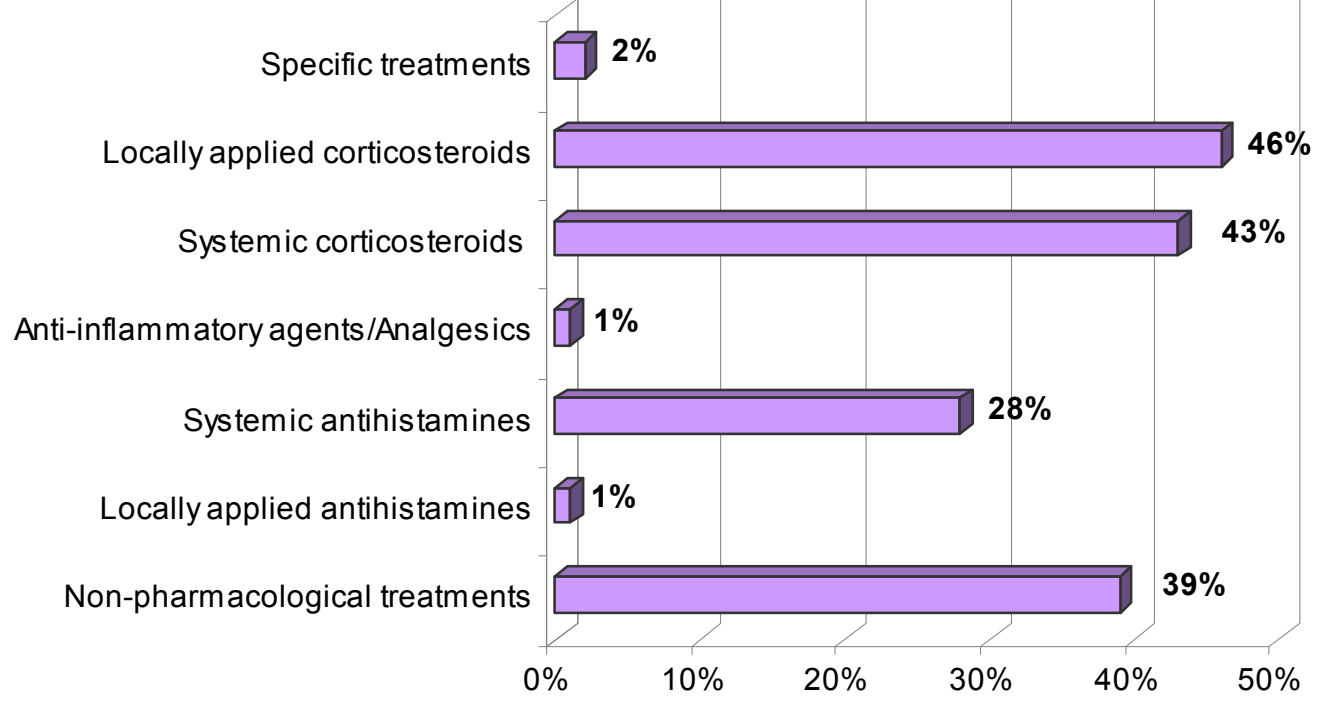

In total, 1,733 jellyfish envenomations in the five years of observation along the relatively short Salento coastline $(215 \mathrm{~km})$ determined an expenditure of over $€ 391,600$ to the Italian National Health Service [33]. This figure does not include the expenses sustained by non-targeted envenomated beachgoers for self-medication purchased directly in pharmacy (topical treatments, bendages) or of bills issued by professional medical consultants. 


\section{Discussion}

Jellyfish envenomations represent an emerging environmental health issue impacting mainly a highly sensitive group of seasonal bathers, i.e., young children. Recent estimates suggest 150 million jellyfish stings worldwide every year [15,34], with up to several hundreds of daily reports in tourist areas, therefore a representing a severe threat not only for human health, but also for coastal tourism and sea-based economies.

Jellyfish stings are among the commonest reasons for requesting medical assistance at summer first aid stations along the coasts of Salento (South Italy), an area with high tourist development and frequent occurrence of jellyfish, and the main pathology due to contact with marine organisms.

The number of reported stinging incidents in this study is largely underestimating the actual number of bathers envenomated by jellyfish during the bathing season, since most adult stung bathers do not seek for medical attention by first aid centres and therefore the majority of cases of stings are not reported.

Compared to data of jellyfish occurrence, a decreasing trend of jellyfish stings was observed from 2009 to 2011, when the lowest number of cases was recorded. Conversely, in the same study area dense jellyfish populations has been increasingly reported in the same period, as detected by the citizen science campaign named METEOMEDUSE (Jellyfish forecasting). The decreasing trend of recorded A\&E events at first aid centres due to jellyfish stings can be interpreted as a positive outcome of vast informative campaigns carried out by research projects on jellyfish, with the production and distribution of thousands of posters for the identification of stinging jellyfish. Also guides for prevention and mitigation of stings, fact sheets on the most common jellyfish species, and weekly reports about jellyfish density in the area were published online on the meteomeduse.focus.it website. In the same years, every August the same poster for jellyfish identification was published in the monthly magazine of FOCUS (monthly selling over 400,000 copies in Italy).

The distribution of affected patients revealed the greater incidence of jellyfish envenomations along the Adriatic coast rather than along the Ionian Sea, due to the predominance of northerly winds driving drifted jellyfish towards the Adriatic coast.

In line with previous observations [35], the highest number of stung bathers requiring medical attention belongs to the younger age groups, particularly the $0-10$ and 11-20 age groups. This might be explained because youth are more likely to spend longer time in water, so more exposed to the risk of jellyfish stings. Conversely, it is worth noting that large stinging jellyfish species (e.g., Rhizostoma pulmo) can be observed only at a distance from the shoreline, where younger bathers are usually not encountered. Possibly, the minor sensitivity of the older age groups can be explained by the longer pain tolerance with age [36], which make less probable the need to seek for medical assistance.

In most cases the reported reactions were mild, exclusively local and cutaneous, in line with what has emerged in other epidemiological studies conducted in other parts of the Mediterranean [10,21]. However, several cases were characterized by complications, even including a case of anaphylactic shock. This is quite unusual, given the relatively low threat posed by the typical jellyfish occurring in the Mediterranean sea [11,31]. Previous studies reported only another case of anaphylactic shock from the Greek coast, in the studied period from 1981 to 1984 [19].

Concerning therapeutic methods, there isn't a standard treatment protocol against Mediterranean jellyfish stings because of the lack of general consensus, mainly due to the paucity of clinical trials. 
Nevertheless, available evidence suggests that a unitary protocol cannot be applied for all jellyfish stings, as the nature and action mode of cnidarian venom depends on the source organism [15,20]. In most cases corticosteroids were administered for local or systemic use depending on the seriousness of the symptoms; washing the affected skin is mainly undertaken with ammonia solutions. In some cases the improper uses of alcohol, ice and cold or hot fresh water were reported, treatments to be avoided in that they can cause the firing of nematocysts that have not yet discharged [16,17,20]. Jellyfish stings remain a common form of envenomation, and yet confusion appears to exist in the community as to the correct first aid to apply. The primary line of treatment for the major cause of jellyfish envenomation in the Mediterranean Sea, Pelagia noctiluca, is summarized as follows in short consecutive steps [20]: (i) flushing with seawater, (ii) short application (2') of a baking soda slurry ( $50 \%$ in seawater). This slurry revealed to be an effective tool to block further nematocyst discharge; (iii) removal of residual tentacle pieces using plastic cards and (iv) application of ice packs (5'-15'). If pain persists, a topic analgesic (e.g., lidocaine 3-4\%) and/or hydrocortisone cream can relieve pain and reduce inflammation due to envenomations by $P$. noctiluca and other jellyfish belonging to the same Pelagiidae family (e.g., Chrysaora hysoscella) $[15,20]$.

Local residents represented the majority of treated patients $(65 \%)$, however a large number of envenomations occurred also in non-resident patients, with potentially serious consequences for tourism and for the overall service sector of the economy, associated with tourism. Management plans for tourism promotion should incorporate proactive initiatives such as education to jellyfish issues provided by lifeguards and hotel staff, or the installation of adequate informative signage at popular beaches. Beach closure at time of high-density jellyfish swarms should be planned along Italian coastlines, as recently applied by Spanish beach authorities when hundreds of specimens of a highly venomous jellyfish (P. physalis) were spotted along northern Atlantic coasts [37]. Building capacities to apply integrated monitoring protocols, including the creation of local emergency Task Forces by jellyfish-specific training of coastal managers and water agencies operators, as well as the development of prevention plans against jellyfish impacts, are tasks of the ENPI-CBC MED funded project MED-JELLYRISK (2012-2015) (http://www.jellyrisk.eu).

In the period of study, along the $215 \mathrm{~km}$ of coast observed, 1,733 bathers sought medical treatment for jellyfish stings, with an average incidence of 8.1 cases $/ \mathrm{km}$ of coast. The majority (73\%) of the coastline in Salento is characterized by rocky shores, where attendance of bathers is expected to be lower than at sandy beaches. Also, several localities along the Italian coastline during the period of investigations recorded much denser and more frequent jellyfish outbreaks than on the Salento coastline (data not shown). Although jellyfish do not occur everywhere along the Italian coasts, many tourist hot spots affected by severe jellyfish outbreaks (e.g., Sicily and its minor islands, Sardinia, Tuscan Archipelago and neighbouring areas, Ligurian Sea) are visited by several millions of seasonal tourists. In 2004, over 63 million international tourists travelled to the Italian coastline [38], but only a minority of them (2-2.5\%) reached the Apulia regional coasts. Therefore, the figures obtained for the small Salento peninsula might well underestimate the overall impact of jellyfish along the tourist hot spots along the Italian coasts. Nevertheless, assuming a comparable average incidence of 8.1 cases $/ \mathrm{km}$ of coast throughout Italy, about 40,000 potential cases of jellyfish stings might have occurred along the 4,970 km of Italian bathing coasts in the 2007-2011 period, with a gross estimate of jellyfish direct cost to Italian Health Services of about 2 million Euro/year. 
Besides the cost of first aid services, the socio-economic impact of stinging jellyfish outbreaks in coastal areas can be much higher, as it will include the cost of ongoing treatments and drugs for envenomed bathers, the economic value of disrupted holidays, the change in recreational choices (stung bathers will not return to the same beach until the jellyfish swarm disappear).

Information about jellyfish (occurrence, abundance, species composition) can be therefore crucial in understanding and prevention of jellyfish impacts, by allowing tourists to modify their recreational choices and/or adopt preventive countermeasures (e.g., choice of beach, use of underwater mask, use of skin protective creams). Seaside tourism represents one of the strongest selling points for Italy, hence the social value for jellyfish monitoring and forecasting tools can be large, and it should be coupled to mitigation strategies and policy regulations. Use of anti-jellyfish coastal nets at hot spots of jellyfish proliferations, appropriate training of lifeguards and coastal stakeholders against jellyfish proliferations, development of standardized species-specific protocols of treatment, might be crucial strategies in the future years for the prevention of health hazards and promotion of seaside leisure activities.

\section{Conclusions}

In summary, the data presented here demonstrate that management of jellyfish outbreak phenomena will deserve coordinated research efforts towards a better understanding of underlying ecological mechanisms, together with the adoption of effective primary prevention, mitigation strategies, and appropriate planning of health services at tourist hot spots. In this framework, citizen science campaigns can increase public perception of jellyfish impacts and can represent an important tool towards the reduction of health hazards and the social cost of this emerging phenomenon.

\section{Acknowledgments}

The authors thank Livio Ruggiero, who is responsible for the Laboratory of Climatology, and Gennaro Rispoli, Department of Materials Science at University of Salento, for supplying the meteorological data relative to the Lecce site. Thanks also to the staff of the Health Districts (ASL/LE) for support in data collection. Jennifer Purcell (CONISMA) kindly provided valuable comments. The research leading to these results has received funding from the European Community's Seventh Framework Programme (FP7/2007-2013) for the project VECTORS (http://www.marinevectors.eu), and by the ENPI CBCMED programme for the project MED-JELLYRISK (http://www.jellyrisk.eu). Additional logistic support and use of facilities was obtained from the FP7 EU projects COCONET (http://www.coconet-fp7.eu) and PERSEUS (http://www.perseus-net.eu), and from the Italian flagship project RITMARE (www.ritmare.it).

\section{Author Contributions}

Antonella De Donno, Ferdinando Boero and Stefano Piraino: study design, data analysis and manuscript writing; Adele Idolo and Tiziana Grassi: epidemiological data collection, and analysis, manuscript writing; Francesco Bagordo: study design and revision of the manuscript; Alessandro Leomanni and Francesca Serio performed data acquisition and literature review; Mariarita Canitano 
coordinated the data acquisition; Serena Zampardi and Marcello Guido: data analysis and preparation of illustrations. All authors read and approved the final manuscript.

\section{Conflicts of Interest}

The authors declare no conflict of interest.

\section{References}

1. Brotz, L.; Cheung, W.W.L.; Kleisner, K.; Pakhomov, E.; Pauly, D. Increasing jellyfish populations: Trends in large marine ecosystems. Hydrobiologia 2012, 690, 3-20.

2. Purcell, J.E.; Uye, S.I.; Lo, W.T. Anthropogenic causes of jellyfish blooms and direct consequences for humans: A review. Mar. Ecol. Prog. Ser. 2007, 350, 153-174.

3. Purcell, J.E. Jellyfish and ctenophore blooms coincide with human proliferations and environmental perturbations. Ann. Rev. Mar. Sci. 2012, 4, 209-235.

4. Boero, F.; Bouillon, J.; Gravili, C.; Miglietta, M.P.; Parsons, T.; Piraino, S. Gelatinous plankton: Irregularities rule the world (sometimes). Mar. Ecol. Prog. Ser. 2008, 356, 299-310.

5. Lynam, C.P.; Hay, S.J.; Brierley, A.S. Interannual variability in abundance of North Sea jellyfish and links to the North Atlantic Oscillation. Limnol. Oceanogr. 2004, 49, 637-643.

6. Lynam, C.P.; Hay, S.J.; Brierley, A.S. Jellyfish abundance and climatic variation: Contrasting responses in oceanographically distinct regions of the North Sea, and possible implications for fisheries. J. Mar. Biol. Assoc. UK 2005, 85, 435-450.

7. Purcell, J.E. Climate effects on formation of jellyfish and ctenophore blooms. J. Mar. Biol. Assoc. UK 2005, 85, 461-476.

8. Attrill, M.J.; Wright, J.; Edwards, M. Climate-related increases in jellyfish frequency suggest a more gelatinous future for the North Sea. Limnol. Oceanogr. 2007, 52, 480-485.

9. Lynam, C.P.; Lilley, M.K.S.; Bastian, T.; Doyle, T.K.; Beggs, S.E.; Hays, G.C. Have jellyfish in the Irish Sea benefited from climate change and overfishing? Glob. Change Biol. 2011, 17, 767-782.

10. Mariottini, G.L.; Pane, L. Mediterranean jellyfish venoms: A review on scyphomedusae. Mar. Drugs 2010, 8, 1122-1152.

11. Boero, F. Review of Jellyfish Blooms in the Mediterranean and Black Sea. In General Fisheries Commission for the Mediterranean, Studies and Reviews 2013; FAO: Rome, Italy, 2013.

12. Frazão, B.; Vasconcelos, V.; Antunes, A. Sea anemone (Cnidaria, Anthozoa, Actiniaria) toxins: An overview. Mar. Drugs 2012, 10, 1812-1851.

13. Burnett, J.W. Medical aspects of jellyfish envenomation: Pathogenesis, case reporting and therapy. Hydrobiology 2001, 451, 1-9.

14. Mariottini, G.L.; Pane, L. Cytotoxic and cytolytic cnidarian venoms. A review on health implications and possible therapeutic applications. Toxins 2014, 6, 108-151.

15. Cegolon, L.; Heymann, W.C.; Lange, J.H.; Mastrangelo, G. Jellyfish stings and their management: A review. Mar. Drugs 2013, 11, 523-550. 
16. Birsa, L.M.; Verity, P.G.; Lee, R.F. Evaluation of the effects of various chemicals on discharge of and pain caused by jellyfish nematocysts. Comp. Biochem. Physiol. C Toxicol. Pharmacol. 2010, $151,426-430$.

17. Ward, N.T.; Darracq, M.A.; Tomaszewski, C.; Clark, R.F. Evidence-based treatment of jellyfish stings in North America and Hawaii. Ann. Emerg. Med. 2012, 60, 399-414.

18. Auerbach, P.S. Envenomations from jellyfish and related species. J. Emerg. Nurs. 1997, 23, 555-565.

19. Vlachos, P.; Kontos, P. Epidemiology and therapeutic methods of "jellyfish" poisoning in Greece. In Jellyfish blooms in the Mediterranean, Proceedings of the II Workshop on Jellyfish in the Mediterranean Sea, Trieste, Italy, 2-5 September 1987; pp. 302-308.

20. Treatment of Mediterranean Jellyfish Envenomations. Available online: https://www.facebook. $\mathrm{com} / \mathrm{media} / \mathrm{set} /$ ?set $=\mathrm{a} .178467932333678 .1073741837 .144960982351040 \&$ type $=1 \quad$ (accessed on 7 February 2014).

21. Mariottini, G.L.; Giacco, E.; Pane, L. The mauve stinger Pelagia noctiluca (Forsskål, 1775). Distribution, ecology toxicity and epidemiology of stings. Mar. Drugs 2008, 6, 496-513.

22. Canepa, A.; Fuentes, V.; Sabatès, A.; Piraino, S.; Boero, F.; Gili, J.M. Pelagia noctiluca in the Mediterranean Sea. In Jellyfish Blooms. Pitt, K.A., Lucas, C.H., Eds.; Springer: Berlin, Germany, 2014; pp. 237-266.

23. Deidun, A.; Arrigo, S.; Piraino, S. The westernmost record of Rhopilema nomadica (Galil, 1990) in the Mediterranean-Off the Maltese Islands. Aquat. Invasions 2011, 6, S99-S103.

24. Yahia, M.N.D.; Kefi-Daly Yahia, O.; Gueroun, S.; Aissi, M.; Deidun, A.; Fuentes, V.; Piraino, S. The invasive tropical scyphozoan Rhopilema nomadica Galil, 1990 reaches the Tunisian coast of the Mediterranean Sea. BioInvasions Rec. 2013, 2, 319-323.

25. Kokelj, F.; Lo Brutto, R.; Boccucci, N. Epidemiological study of human injuries following jellyfish stings in the Gulf of Trieste. Contact Dermat. 1999, 41, 349-350.

26. Malej, A.; Vukovic, A. Some Data on the Occurrence and Biology of the Scyphomedusa Pelagia noctiluca in the Gulf of Trieste, and the Impact of Jellyfish Swarming on Human Activities. In Workshop on Jellyfish Blooms in the Mediterranean; UNEP: Athens, Greece, 31 October4 November 1984; pp. 89-94.

27. Maretic, Z. The Bloom of Jellyfish Pelagia noctiluca along the Coasts of Pula and Istria 19771983, with Special Reference to Epidemiology, Clinics and Treatment. In Workshop on Jellyfish Blooms in the Mediterranean; UNEP: Athens, Greece, 31 October-4 November 1984; pp. 83-87.

28. Maretic, Z.; Matic-Piantanida, D.; Ladavac, J. The bloom of the jellyfish Pelagia noctiluca in the Mediterranean and Adriatic and its impact on human health. In Jellyfish blooms in the Mediterranean, Proceedings of the II Workshop on Jellyfish in the Mediterranean Sea, Trieste, Italy, 2-5 September 1987; pp. 260-267.

29. Scarpa, C. On Skin Injuries Provoked by Coelenterata And Echinodermata. In Workshop on Jellyfish Blooms in the Mediterranean; UNEP: Athens, Greece, 31 October-4 November 1984; pp. 95-97. 
30. Carli, A.; Mariottini, G.L.; Pane, L. Ecological and Medical Aspects of Jellyfish Poisoning. In Epidemiological Studies Related to the Environmental Quality Criteria for Bathing Waters, Shellfish-Growing Waters and Edible Marine Organisms; UNEP: Athens, Greece, 1995; pp. 1-21.

31. De Donno, A.; Idolo, A.; Bagordo, F. Epidemiology of jellyfish stings reported to summer health centres in the Salento peninsula (Italy). Contact Dermat. 2009, 60, 330-335.

32. Conoscere La Provincia Di Lecce. Available online: http://www3.provincia.le.it/statistica/ (accessed on 11 March 2013).

33. Ministero Della Salute. Progetto Mattoni SSN. Available online: http://www.mattoni.salute.gov.it/ (accessed on 11 March 2013).

34. Boulware, D.R. A randomized controlled field trial for the prevention of jellyfish stings with a topical sting inhibitor. J. Travel Med. 2006, 13, 166-171.

35. Forrester, M.B. Epidemiology of jellyfish stings reported to poison centers in Texas. Hum. Exp. Toxicol. 2006, 25, 183-186.

36. Schmitz, A.K.; Vierhaus, M.; Lohaus, A. Pain tolerance in children and adolescents: Sex differences and psychosocial influences on pain threshold and endurance. Eur. J. Pain 2012, 17, 124-131.

37. Govan, F. Deadly jellyfish close beaches in Spain. Available online: http://www.telegraph.co.uk/ news/worldnews/europe/spain/9490141/Deadly-jellyfish-close-beaches-in-Spain.html (accessed on 17 February 2014).

38. Quintiliani, F. International tourism in the coastal regions of five Mediterranean countries. Tour. Anal. 2009, 14, 353-373.

(C) 2014 by the authors; licensee MDPI, Basel, Switzerland. This article is an open access article distributed under the terms and conditions of the Creative Commons Attribution license (http://creativecommons.org/licenses/by/3.0/). 